3. A 4-year-old Cavalier King Charles Spaniel presented with an acute onset of right head tilt and left-sided proprioceptive deficits. What is the neuroanatomical localization?
(A) Peripheral vestibular localization

(B) Central vestibular localization - brainstem

(C) Right cranial cervical localization

(D) Paradoxical vestibular localization

\section{What do you need to know about brain MRI?}

\section{Tim Trevail}

MRI is widely regarded as the imaging modality of choice in the diagnostic imaging evaluation of the neurological patient. However, an MRI scan is a very costly investigative tool and clinicians choosing this as the next appropriate test should be aware of the benefits and limitations of MRI compared with alternative imaging modalities. Benefits:

- Exquisite soft tissue detail delivers outstanding brain and spinal cord definition

- Cross-sectional imaging technique, takes away superimposition noted on radiographs

- Ability to give accurate information on tissue content such as haemorrhage or free fluid.

Limitations:

- Expensive

- Some safety considerations given the high magnetic field; staff need to be appropriately trained to avoid catastrophic incidents

- Bone imaging inferior to computed tomography for determining fractures and for surgical planning

- Relatively slow image acquisition time.

When compared to the other imaging modalities, MRI may be considered somewhat complicated. This is in part due to the complex physics associated with image acquisition but also due to the huge variety of sequences available. The most important are listed below and will be discussed during the lecture.

- T1-weighted: produced using short time to echo (TE) and time to repetition (TR) parameters and one of the staple sequences in MRI. Fluid appears dark (hypointense) and fat bright (hyperintense) on these images. T1-weighted images give good anatomical information. This sequence can be combined with the use of an intravenous gadolinium-based contrast agent, where contrast uptake indicating vascularization appears bright

- T2-weighted: produced using long TE and TR parameters and again a staple sequence. Fluid and fat both appear bright (hyperintense) on this sequence. Within the neural parenchyma, due to oedema being associated with most lesions in the brain and spinal cord, lesions are often best detected on T2-weighted images

- Fluid attenuating inversion recovery (FLAIR): this technique nulls the signal from free fluid, allowing the differentiation of free fluid (such as within a cyst or the ventricles) from oedema

- Short tau inversion recovery (STIR): this technique nulls the signal from fat and as such allows identification of oedema and reaction within paravertebral tissues, muscles and bone marrow - Gradient echo/T2*: a particularly useful sequence to identify the presence of haemorrhage or mineralization within tissues due to the presence of magnetic susceptibility effects. Gradient echo images are also good for evaluating bone

- Diffusion weighting imaging (DWI): a technique used to evaluate the diffusion of molecules (mainly water) through tissues and is very useful where cellular swelling is present (which reduces diffusion) such as in acute infarction.

Interpretation of brain MRI requires an understanding of the tissue characteristics defined by the above sequences and an understanding of the possible pathologies that may be encompassed when imaging the brain, in addition to an in-depth knowledge of brain anatomy. Several features of common brain pathologies will be discussed during this lecture.

\section{KEY LEARNING OBJECTIVES}

Understand the limitations and benefits of magnetic resonance imaging (MRI) for imaging the brain of the neurological patient

- Know what sequences are available for brain MRI, what they tell you and when to use them

- Know the interpretive principles of brain MRI and be able to identify the more common pathologies of the brain in dogs and cats

\section{MULTIPLE CHOICE QUESTIONS}

1. Which of the following features is commonly identified in extra-axial mass lesions?
(A) Poor contrast enhancement
(B) Haemorrhage
(C) Rim enhancement
(D) A dural tail 


\section{AVP: Diagnostic imaging}

2. Which of the following sequences is routinely used to aid differentiation between suspected ischaemic and neoplastic lesions?
(A) DWI
(B) T1-weighted spin echo post contrast
(C) FLAIR
(D) Gradient echo

3. In which of the following instances should MRI be the initial imaging modality of choice?

(A) To evaluate the cause of seizures in a 6-month-old female entire Miniature Schnauzer with postprandial bile acids of $90 \mu \mathrm{mol} / \mathrm{l}$
(B) To evaluate the cause of seizures in a 7-year-old female neutered Golden Retriever with a recent history of change in behaviour

(C) To evaluate the cause of seizures in a 9-year-old female neutered West Highland White Terrier with a normal neurological examination who had a pacemaker placement for sick sinus syndrome 3 years ago

(D) To evaluate the cause of seizures in an 8-year-old female neutered Cocker Spaniel with recently diagnosed mammary carcinomas

\section{Leaky pipes or blocked drains: urinary contrast studies in 2020}

\section{Anna Newitt}

In cases of dysuria, a urethral obstruction is of concern, although there may be apparent dysuria in severe cases of bladder irritation when the bladder is in fact empty. The main differentials for urethral obstruction are uroliths, severe urethritis/urethral stricture or bladder neck/ urethral neoplasia. In a male dog, prostatic disease is also a consideration. As uroliths are the most frequent differential, plain radiographs are an important first diagnostic step in dysuria. Lumbar spine or pelvic metastasis of prostatic neoplasia or other pelvic masses associated with extrinsic urethral compression may also be identified with plain radiographs.

If plain radiographs demonstrate bladder distension, although are otherwise normal, then patient signalment and clinical suspicion are the most useful guides in further evaluation. Ultrasonography is extremely useful for evaluation of the bladder and the bladder neck and the abdominal portion of the urethra can also be assessed with ultrasonography; identification of neoplasia at this site is therefore easiest and best with ultrasonography. A retrograde urethrogram is a very helpful technique, although is easier to perform and interpret in male animals. A urethral rupture, stricture or obstruction of other origin can all be identified with a well performed retrograde urethrogram; occasionally urethral spasm may mimic urethral luminal narrowing and a repeated retrograde urethrogram is frequently needed to assess if these are consistent findings.

In cases of incontinence, complete evaluation will include biochemistry, haematology and urinalysis for evaluation of any underlying renal disease or other causes for polyuria, which may result in overflow incontinence.
Abdominal ultrasonography is superior to an IVU for initial assessment of renal parenchyma and for assessment of renal pelvic/ureteric dilation. If the ureters are dilated then these may be followed either to the insertion in the urinary bladder, urethra or elsewhere. If the ureters are not dilated or if the bladder neck is caudally located within the pelvic canal then an IVU can be useful. It is more technically difficult to obtain a good-quality radiographic IVU than with CT; with radiographic studies, repeated radiographs at frequent intervals are needed to assess termination within the urinary bladder. A prior pneumocystogram is also very helpful for assessing ureteric insertion. CT assessment does not require a pneumocystogram and removes the complication of superimposed structures creating artefact.

\section{KEY LEARNING OBJECTIVES}

- Plain radiographs are often of limited value in assessment of incontinence, although are an important first diagnostic step in dysuria

- Ultrasonography is very helpful for assessing renal parenchyma and renal pelvic dilation; with experience, it is frequently possible to follow dilated ureters and identify sites of obstruction

- Decision for intravenous urogram (IVU), ultrasonography or computed tomography (CT) evaluation of the ureters will depend on availability of the modality; a caudal bladder neck may mean that sonographic evaluation of ureteric insertions is extremely difficult

\section{MULTIPLE CHOICE QUESTIONS}

1. Which the following is not associated with filling defects in a positive-contrast urethrogram?

(A) Benign prostatic hyperplasia

(B) Air bubbles 